\title{
Tromboembolismo venoso em Cirurgia Plástica: protocolo de prevenção na Clínica Ivo Pitanguy
}

\section{Venous thromboembolism in Plastic Surgery: prevention protocol at Ivo Pitanguy Clinic}

\author{
Rita Azevedo de Paiva ${ }^{1}$ \\ Ivo PitanguY ${ }^{2}$ \\ Natale F. Gontijo \\ DE Amorim $^{3}$ \\ RALF Berger ${ }^{4}$ \\ HAZEL DE \\ ANDRADE SHDICK ${ }^{4}$ \\ Thiago Ayres Holanda ${ }^{4}$
}

Trabalho realizado no Instituto Ivo Pitanguy, Rio de Janeiro, RJ, Brasil.

Artigo submetido pelo SGP (Sistema de Gestão de Publicações) da RBCP.

Artigo recebido: 14/8/2010 Artigo aceito: 18/10/2010

\begin{abstract}
RESUMO
Introdução: A trombose venosa profunda (TVP) e o seu desfecho imediato mais grave, o tromboembolismo pulmonar (TEP), são complicações de incidência elevada em pacientes hospitalizados e principalmente naqueles submetidos à cirurgia. Objetivo: Apresentar o protocolo de profilaxia de Tromboembolismo (TEV) da Clínica Ivo Pitanguy, mostrando a incidência de TEV no Serviço antes e após a introdução deste protocolo, assim como a incidência de hematomas, no período de cinco anos. Método: Estudo de revisão dos prontuários dos pacientes operados no período de julho de 2004 a maio de 2009. Comparou-se a incidência de eventos trombóticos antes e após a introdução do protocolo, assim como a incidência de hematomas. Resultados: Foram analisados 1700 prontuários. Desde a introdução do protocolo, não ocorreu nenhum caso de tromboembolismo venoso, em 711 pacientes operados. A incidência de hematomas com necessidade de revisão cirúrgica foi reduzida, provavelmente pelo controle pressórico mais rigoroso após a introdução do protocolo. Conclusão: O protocolo tem se mostrado de utilização simples e efetiva. Houve redução da incidência de TEV e hematomas a partir de sua introdução.
\end{abstract}

Descritores: Tromboembolia venosa. Embolia pulmonar. Cirurgia plástica.

\begin{abstract}
Background: The deep vein thrombosis (DVT) and its most dangerous outcome, the pulmonary embolism (PE), are complications of high incidence in hospitalized patients and in those submitted to surgery. Objective: This study presents the guideline to prevention of thromboembolism at Ivo Pitanguy Clinic and its incidence before and after the introduction of this protocol, in a period of five years. We also relate the incidence of hematoma before and after the beginning of the protocol. Methods: The study consists of the revision of the medical records of all patients operated between July 2004 and May 2009. We compared the incidence of thromboembolic events before and after the beginning of the protocol as well as the incidence of hematomas that were submitted to revision at the surgery room. Results: Analyzing 1700 medical records, since the introduction of the guideline of prevention of VTE, there is no case of VTE in 711 operated patients. The incidence of reoperated hematomas at the surgery room was reduced, maybe because there was a more rigorous control of the blood pression after the introduction of the guideline. Conclusion: The guideline has shown itself practical and simple use. There were reduction in cases of VTE and hematomas after its introduction.
\end{abstract}

Keywords: Venous thromboembolism. Pulmonary embolism. Plastic surgery.

1. Mestre em Medicina Intensiva pela Universidade Federal do Rio de Janeiro; Chefe do Serviço de Terapia Intensiva Perioperatória do Instituto Ivo Pitanguy. Professora Colaboradora dos cursos de Pós-Graduação Médica em Cirurgia Plástica pela Pontifícia Universidade Católica do Rio de Janeiro (PUC-RJ) e pelo Instituto de Pós-Graduação Médica Carlos Chagas, Rio de Janeiro, RJ, Brasil.

2. Patrono da Sociedade Brasileira de Cirurgia Plástica (SBCP); Professor Titular do Curso de Pós-Graduação Médica em Cirurgia Plástica pela PUC-RJ e pelo Instituto de Pós-Graduação Médica Carlos Chagas, Rio de Janeiro, RJ, Brasil. Membro da Academia Nacional de Medicina e Academia Brasileira de Letras.

3. Membro Titular da Sociedade Brasileira de Cirurgia Plástica; Professora Adjunta dos cursos de Pós-Graduação Médica em Cirurgia Plástica pela PUC-RJ e pelo Instituto de Pós-Graduação Médica Carlos Chagas. Pesquisadora-chefe do Instituto Ivo Pitanguy. Cirurgiã Plástica da Equipe do Prof. Ivo Pitanguy.

4. Cirurgião Geral; Pós-graduando do Instituto Ivo Pitanguy, Rio de Janeiro, RJ, Brasil. 


\section{INTRODUÇÃO}

O termo tromboembolismo venoso (TEV) refere-se ao variado espectro de apresentação clínica, que varia desde a trombose venosa profunda à embolia pulmonar. Esta última, por sua vez, é uma das causas de morte hospitalar mais evitáveis, apesar de responder por mais de 200.000 mortes anuais somente nos Estados Unidos ${ }^{1}$.

A trombose venosa profunda (TVP) e o seu desfecho imediato mais grave, o tromboembolismo pulmonar (TEP), são componentes da entidade nosológica conhecida como TEV e são complicações relativamente comuns nos pacientes hospitalizados ${ }^{2}$, principalmente naqueles submetidos à cirurgia.

Recentemente, este tema tem recebido mais atenção dentro da Cirurgia Plástica, visando à maior segurança na execução de procedimentos ${ }^{3}$. De acordo com dados da American Society of Plastic Surgeons (ASPS), ocorrem 18.000 casos/ano de TVP em pacientes submetidos à cirurgia plástica. $\mathrm{O}$ aumento do número de cirurgias plásticas realizadas e a maior frequência de cirurgias combinadas, aumentando a possibilidade de complicações, são aspectos que tornam vulnerável o cirurgião plástico. As complicações advindas de cirurgias eletivas estéticas têm repercussão mais grave tanto no âmbito familiar quanto no hospitalar, havendo menor complacência para sua ocorrência. Na literatura, existem poucas publicações sobre a incidência de TEV em Cirurgia Plástica, no Brasil e no mundo, assim como protocolos sugeridos para sua prevenção $0^{4}$.

O objetivo deste trabalho é apresentar o protocolo de profilaxia de TEV da Clínica Ivo Pitanguy, analisar a incidência de TEV antes e após sua introdução bem como a incidência de hematomas.

\section{MÉTODO}

Foi realizado estudo retrospectivo por meio da revisão de prontuários dos pacientes operados nos últimos cinco anos na Clínica Ivo Pitanguy, no período de julho de 2004 e maio de 2009. O protocolo de prevenção de TEV foi introduzido no Serviço em maio de 2007.

O protocolo instituído combina o modelo de risco de Caprini et al. ${ }^{5}$ e de Davison et al. ${ }^{1}$, com algumas modificações. Dentre suas limitações estão as descrições indefinidas sobre cirurgia maior e cirurgia menor e o fato de não terem sido incluídos pacientes submetidos à cirurgia plástica. Consideramos, em nosso estudo, cirurgias de maior porte aquelas que duram mais de 60 minutos.

Os pacientes candidatos à cirurgia em nosso Serviço são submetidos a avaliação clínica rigorosa pelo Serviço de Terapia Intensiva, onde é estabelecido o risco cirúrgico. Neste momento, o paciente é estratificado quanto ao seu risco individual para ocorrência de TEV. Cada item dos fatores de risco confere uma pontuação ao paciente, que é cumulativa. Ao final da avaliação, os pontos são somados, gerando um escore, e o paciente é enquadrado em uma das categorias de risco: baixo, moderado, alto ou altíssimo. O protocolo é apresentado no Quadro 1.

Como podemos observar no Quadro 1, os pacientes são avaliados quanto aos fatores a que são expostos e aos fatores predisponentes, gerando uma pontuação que determinará a conduta profilática a ser adotada.

A deambulação precoce é estimulada em todos os pacientes, isto é, deambulação na noite do dia da cirurgia. $\mathrm{O}$ uso da meia elástica é indicado para todos os pacientes e é iniciado no transoperatório e mantido por uma semana. A compressão pneumática, quando indicada, é iniciada após a indução anestésica e mantida até o dia seguinte. Nos pacientes com pontuação elevada (acima de 4 pontos) compatível com risco altíssimo para TEV, a HBPM é administrada 12 horas após o término da cirurgia, $40 \mathrm{mg}$ por via subcutânea, podendo ser reaplicada após 24 horas em caso de dificuldade de deambulação. A compressão pneumática intermitente também é mantida nestes casos.

\section{RESULTADOS}

Foram realizadas 1700 cirurgias na Clínica Ivo Pitanguy no período estudado (julho de 2004 a maio de 2009). Neste total, $22,6 \%(\mathrm{n}=384)$ dos pacientes eram do sexo masculino e $77,4 \%(n=1316)$, do sexo feminino. Do total de cirurgias realizadas, $47 \%(n=796)$ foram combinadas e $53 \%(n=904)$ foram procedimentos únicos. Estes dados podem ser observados na Figura 1.

A idade média dos pacientes operados foi 43,8 anos, predominando os pacientes com menos de 40 anos (40\%), seguidos pelos pacientes com idade entre $40-60$ anos (38\%) e por último aqueles maiores de 60 anos $(20 \%)$, como é demonstrado na Figura 2.

Os tipos de anestesias empregados foram divididos entre sedação, anestesia local, anestesia geral e bloqueios medulares. A anestesia mais prevalente foi a geral $(74 \%, \mathrm{n}=1249)$, seguida pela local com sedação $(21 \%, \mathrm{n}=357)$ e local simples $(4 \%, \mathrm{n}=62)$.

No período estudado de 59 meses, foram realizadas 1700 cirurgias, sendo 989 antes da introdução do protocolo de prevenção de TEV e 711 após a introdução do mesmo. A divisão entre os tipos de cirurgias realizadas neste período está registrada na Tabela 1.

A Tabela 2 ilustra a taxa de hematomas que ocorreram neste período em diferentes tipos de procedimentos, antes e após o início do protocolo e que necessitaram de reoperação. Não foram incluídos nesta análise os casos que foram drenados no leito.

No período de julho de 2004 a abril de 2007, foram realizadas 989 cirurgias sem a realização do protocolo de 


\begin{tabular}{|c|c|c|c|}
\hline \multicolumn{4}{|c|}{ Quadro 1 - Fatores de risco TVP. } \\
\hline 1 Ponto & 2 Pontos & 3 Pontos & 5 Pontos \\
\hline Pequena cirurgia $(<1 \mathrm{~h})$ & Grande cirurgia $(>1 \mathrm{~h})$ & IAM prévio & História de fratura de pernas ou quadril \\
\hline Trabalho em pé mais que $6 \mathrm{~h}$ por dia & Acesso venoso profundo & ICC & Paresia/plegia membros inferiores \\
\hline Viagem $>6 h<3$ dias & Gesso/ tala $>72 \mathrm{~h}$ & Sepse & Trauma físico recente \\
\hline \multicolumn{4}{|l|}{ Terapia de reposição hormonal/ ACHO } \\
\hline \multicolumn{4}{|l|}{ PASSO 1 - TOTAL: ___ Pontos } \\
\hline \multicolumn{4}{|c|}{ PREDISPONENTES } \\
\hline Puerpério < 1 mês (1 pt) & \multicolumn{2}{|c|}{ Doença inflamatória intestinal (1 pt) } & Doenças mieloproliferativas \\
\hline Insuf venosa/edema MMII (1 pt) & \multicolumn{2}{|c|}{ Doenças reumatológicas (1 pt) } & Trombocitopenia induzida pela heparina \\
\hline Obesidade - IMC >27 (1 pt) & & & Hiperviscosidade \\
\hline \multicolumn{4}{|l|}{ Neoplasias (2 pts) } \\
\hline \multicolumn{4}{|l|}{ ACHO: Anticoncepcional hormonal oral } \\
\hline \multicolumn{4}{|l|}{ PASSO 2 - TOTAL: __ Pontos } \\
\hline \multicolumn{4}{|c|}{ PASSO 3 - Total da Pontuação nos Passos $1+2$ : ___ Pontos } \\
\hline \multicolumn{4}{|l|}{ PASSO 4 - RECOMENDAÇÃO: } \\
\hline$>4$ pontos & Risco Altíssimo & \multicolumn{2}{|c|}{$\mathrm{ME}+\mathrm{CPI}+\mathrm{HBPM}$} \\
\hline
\end{tabular}

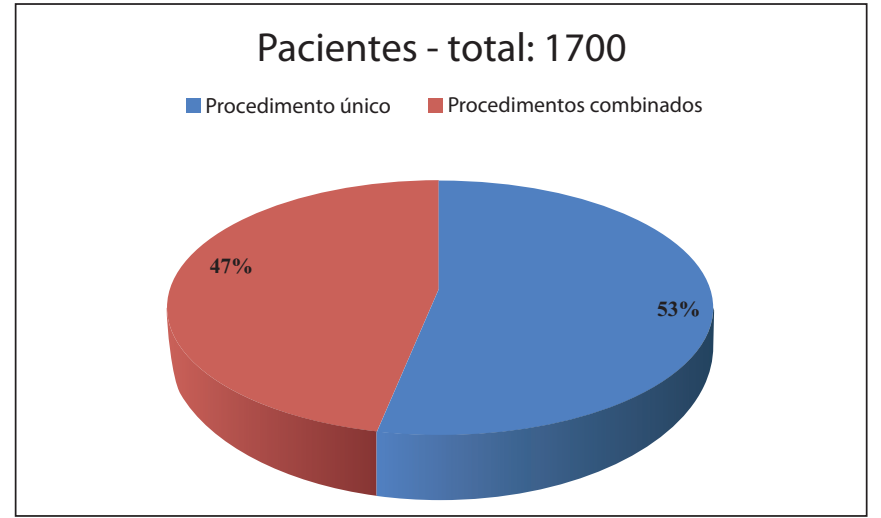

Figura 1 - Percentual de procedimentos únicos ou combinados. prevenção. Ocorreram 8 casos de hematoma com necessidade de abordagem cirúrgica. De maio de 2007 a maio de 2009, já com o estabelecimento do protocolo, foram realizadas 711 cirurgias com ocorrência de 4 casos de hematoma.

Antes da introdução do protocolo de prevenção de TEV, no período estudado de 34 meses, foram realizadas 989 cirurgias e ocorreram dois casos de TEV. O primeiro caso ocorreu em paciente do sexo feminino, de 25 anos, que realizou cirurgia combinada (rinoplastia e implante mamário). Houve ocorrência de TEP no sétimo dia de pós-operatório. Na história clínica, a referida paciente havia realizado viagem aérea de 11 horas, 72 horas antes da cirurgia, e usava anticoncepcional oral.

O segundo caso ocorrido foi em paciente do sexo 


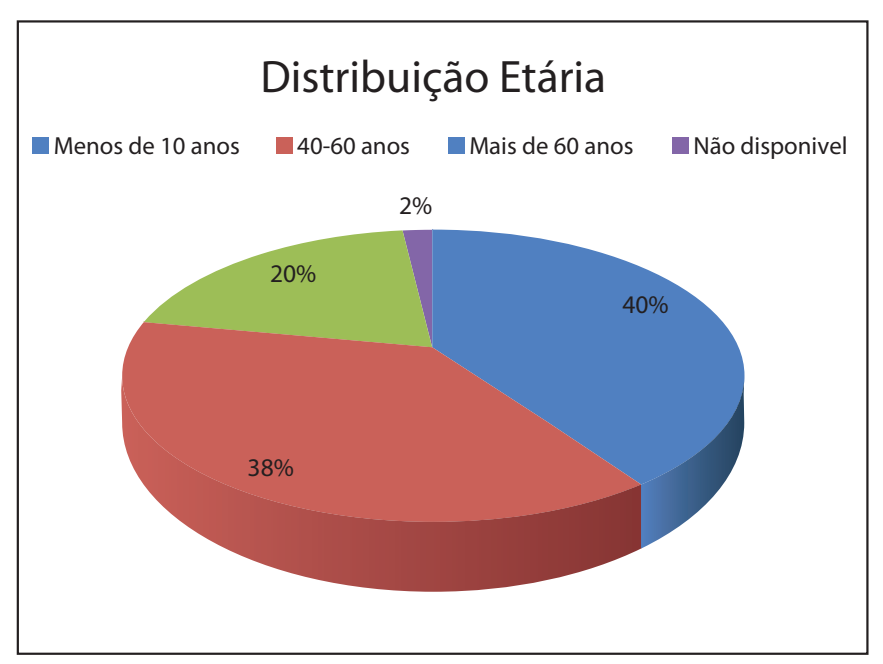

Figura 2 - Faixa etária dos pacientes operados.

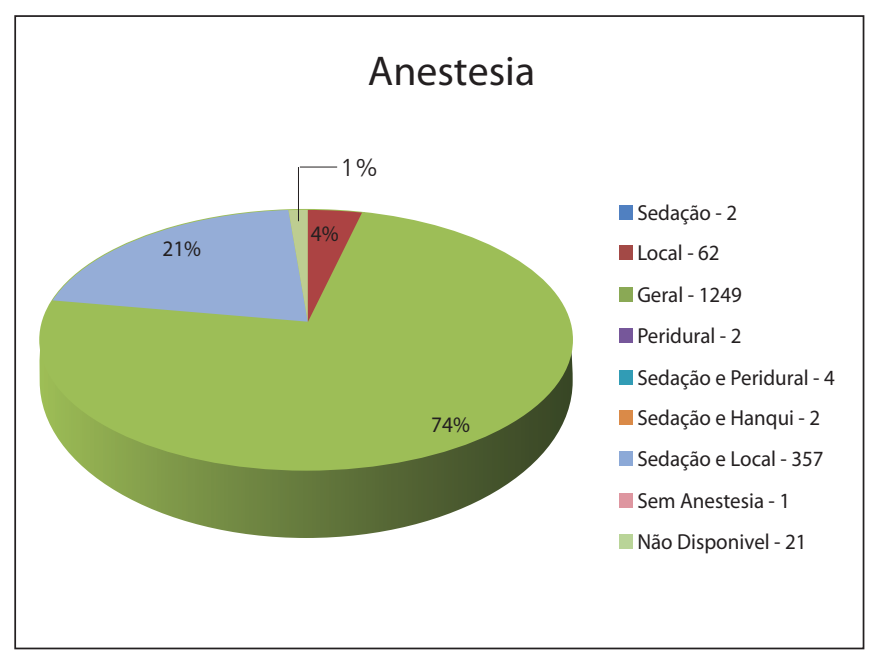

Figura 3 - Tipos de anestesias.

Tabela 1 - Tipos de cirurgias antes e após a introdução do protocolo.

\begin{tabular}{|c|c|c|c|}
\hline Cirurgia & Julho 2004 a Abril 2007 & Maio 2007 a Maio 2009 & TOTAL \\
\hline & Sem Protocolo & Com Protocolo & Total \\
\hline Intervalo Tempo & 34 meses & 25 meses & 59 meses \\
\hline Facelift & 311 & 173 & 484 \\
\hline Video Frontal & 8 & 4 & 12 \\
\hline$\underline{B l e f a r o p l a s t i a ~}$ & 282 & 156 & 438 \\
\hline$\underline{\text { Peeling }}$ & 45 & 23 & 68 \\
\hline Lóbulo & 22 & 11 & 33 \\
\hline Otoplastia & 16 & 8 & 24 \\
\hline Rinoplastia & 165 & 64 & 229 \\
\hline Lipoaspiração & 297 & 150 & 447 \\
\hline Lipoaspiração > $2000 \mathrm{ml}$ & 69 & 54 & 123 \\
\hline Lipoenxertia & 66 & 54 & 120 \\
\hline Mama Redução/ Mastopexia & 86 & 62 & 148 \\
\hline Implante mama & 152 & 93 & 245 \\
\hline Abdominoplastia & 74 & 75 & 149 \\
\hline Lift Braquial & 14 & 14 & 28 \\
\hline Lift Crural & 7 & 3 & 10 \\
\hline Boca & 10 & 4 & 14 \\
\hline Cantoplastia & 68 & 20 & 88 \\
\hline Implante Capilar & 14 & 25 & 39 \\
\hline Prótese Mento & 20 & 2 & 22 \\
\hline Prótese Glúteo & 2 & 1 & 3 \\
\hline Outras Próteses & 3 & 1 & 4 \\
\hline Ninfoplastia & 3 & 2 & 5 \\
\hline Reparadora/ Outras & 459 & 237 & 696 \\
\hline Total & $\mathrm{n}=989$ & $\mathrm{n}=711$ & $\mathrm{n}=1700$ \\
\hline
\end{tabular}




\begin{tabular}{l|c|c}
\multicolumn{3}{c}{ Tabela 2 - Hematoma com abordagem no centro cirúrgico. } \\
\hline $\begin{array}{l}\text { Cirurgia em que } \\
\text { ocorreu hematoma }\end{array}$ & Antes Protocolo & Após Protocolo \\
\hline Facelift & $1,92 \%(n=6 / 311)$ & $1,71 \%(n=3 / 173)$ \\
\hline Implante mamário & $0,65 \%(n=1 / 152)$ & $0 \%(n=0 / 93)$ \\
\hline $\begin{array}{l}\text { Mamoplastia } \\
\text { redutora }\end{array}$ & $1,16 \%(n=1 / 186)$ & $1,61 \%(n=1 / 62)$ \\
\hline Total & $n=8$ & $n=4$ \\
\hline
\end{tabular}

masculino, de 48 anos, que realizou abdominoplastia, também com história de viagem aérea longa ( 8 horas), 48 horas antes da cirurgia. Este paciente apresentou quadro de TVP no quarto dia de pós-operatório.

\section{DISCUSSÃO}

O trombo venoso é composto predominantemente de hemácias e fibrina com composição variável de leucócitos e plaquetas. A formação, crescimento e dissolução deste trombo dependem de um balanço entre estímulo trombogênico e mecanismos protetores ${ }^{6}$. A busca do entendimento dos mecanismos fisiopatológicos envolvidos com o tromboembolismo data de 1859, quando o patologista alemão Rudolf Virchow descreveu os três maiores fatores que ele acreditava serem responsáveis por tal fenômeno: $1^{\circ}-$ a estase venosa causada por mudança no volume ou fluxo sanguíneo; $2^{\circ}-0$ dano ao endotélio vascular por inflamação ou injúria; $3^{\circ}$ - o estado de hipercoagulabilidade ${ }^{7}$. Dentre os fatores protetores, destacamos a inativação de fatores de coagulação por inibidores circulantes (antitrombina III, alfa-1 antitripsina, proteína $\mathrm{C}$ ativada, por exemplo), o clearance de fatores coagulantes ativados e complexos de polímeros de fibrina (pelo sistema reticuloendotelial e fígado) e a dissolução da fibrina por enzimas fibrinolíticas do plasma, bem como a digestão da fibrina por leucócitos.

Especificamente no caso da cirurgia, todos os aspectos da tríade de Virchow são exacerbadamente encontrados. A estase venosa é agravada pela imobilização prolongada na mesa cirúrgica, além disso, o estado pró-coagulante pós-cirúrgico, em que a lesão vascular múltipla inicia uma cascata em direção ao bloqueio do sangramento, também atua diminuindo a atividade fibrinolítica. Já foram comprovados os efeitos de várias substâncias (citocinas, hormônios) e a ação celular e molecular nos estados pró-trombóticos, sendo estes ainda objeto de muitos estudos ${ }^{8}$. Outros estados atuam sinergicamente aos fatores básicos citados, estando entre os principais o tabagismo, o diabetes, a hipertensão arterial, a insuficiência venosa (doença vascular periférica) e a gestação, entre outros. $\mathrm{O}$ ato cirúrgico carrega em si outros condicionantes, como tipo de anestesia utilizada (ainda foco de muitas discussões), a duração e extensão da cirurgia, bem como sua localização, e o estado de hidratação do paciente. A imobilidade pós-operatória também tem muita importância, com a incidência de trombose venosa constatada em autopsia significativamente maior nos indivíduos acamados há mais de três dias, sobretudo se acima de 60 anos, e pacientes com paralisia de membro inferior com um risco de trombose quatro a nove vezes mais alto no membro afetado se comparado ao membro contralateral ${ }^{9}$.

A prevenção do TEV tem ocupado um espaço cada vez mais proeminente nos últimos anos, bem como as propostas para a normatização de protocolos a serem adotados. Especificamente na Cirurgia Plástica, onde o dilema no qual o cirurgião plástico se encontra tendo como algoz o tromboembolismo de um lado e complicações pós-operatórias hemorrágicas do outro ${ }^{10}$, o estabelecimento de um guideline se torna indispensável.

A American Society of Plastic Surgery (Sociedade Americana de Cirurgia Plástica), em 1999, publicou resultados de um Grupo de Estudo ("Task Force") organizado pela instituição ${ }^{11}$. Nesta publicação, foram discutidos os fatores de risco e indicadas algumas medidas profiláticas, mas não foi criado um protocolo que pudesse ser repetido nos vários centros, o que ajudaria a comparar pesquisas clínicas e fornecer dados reais sobre a incidência e morbidade na TVP na Cirurgia Plástica ${ }^{4}$.

Em 2001, Caprini et al. ${ }^{5}$ estratificaram o risco de tromboembolismo venoso em pacientes cirúrgicos e não-cirúrgicos.

Em 2004, Davidson et al. ${ }^{1}$ publicaram um artigo sobre a prevenção de TEV em Cirurgia Plástica, apresentando um protocolo de prevenção.

Em 2007, foi iniciado o Serviço de Terapia Intensiva Perioperatória na Clínica Ivo Pitanguy, o qual consiste de atendimento clínico pré-operatório, onde é feito o preparo do paciente para a cirurgia proposta e estabelecido o risco cirúrgico e do acompanhamento trans e pós-operatório por médico intensivista. Foi então estabelecido um protocolo de prevenção de TEV, combinando a estratificação de risco de Caprini et al. ${ }^{5} \mathrm{e}$ o protocolo de Davison et al. ${ }^{1}$, com modificações. Desde então, não foi identificado nenhum caso de TVP ou TEP.

Neste estudo, foi realizada análise retrospectiva de todas as cirurgias realizadas na clínica Ivo Pitanguy, no período de julho de 2004 a junho de 2009, totalizando 59 meses de estudo, o que correspondeu a 1700 cirurgias. Quase metade das cirurgias realizadas $(47 \%)$ foi constituída de procedimentos combinados, o que é prática cada vez mais frequente na Cirurgia Plástica. As cirurgias combinadas têm sido demonstradas como de maior risco para fenômenos tromboembólicos, com destaque aquelas associadas à abdominoplastia ou à lipoaspiração ${ }^{12-14}$. Faz-se necessário, portanto, preparo minucioso deste paciente para a cirurgia, com acurado índice de suspeição para diagnósticos.

O lifting facial é o procedimento isolado mais executado 
na clínica, sendo também bastante frequentes a lipoaspiração e cirurgias de mama e abdome. A anestesia geral foi o procedimento anestésico mais executado (74\%), seguida pela anestesia local com sedação (21\%). A anestesia geral está relacionada a maior incidência de TEV. Segundo estatísticas de 2001 da Sociedade Americana de Cirurgia Plástica, dentre os pacientes submetidos ao lifting facial que apresentaram episódios de tromboembolismo, 83,7\% foram submetidos à anestesia geral, contra $16,3 \%$ daqueles casos realizados com anestesia local e sedação ${ }^{15}$. Nossa casuística, portanto, inclui procedimentos em cirurgia plástica e técnica anestésica associados ao maior risco de TEV. Por tal razão, tornou-se imperiosa a elaboração de protocolo rigoroso de prevenção.

Segundo dados da literatura, a incidência de TEV em Cirurgia Plástica difere de acordo com o tipo de cirurgia realizada, sendo aproximadamente $0,35 \%$ para lifting facial, $1,3 \%$ para reconstrução de mama, 1,4 a $2 \%$ para abdominoplastia, 9,4\% para lipoaspiração circunferencial e 6,6\% para abdominoplastia associada a outra cirurgia ${ }^{16}$

Conforme os dados analisados, a incidência de TEV na Clínica Ivo Pitanguy já se encontrava abaixo da estatística publicada na literatura e ainda ocorreu redução dos índices após a implantação do protocolo.

As taxas de incidência de hematoma, da mesma forma, também variam de acordo com a cirurgia realizada. As mamoplastias com inclusão de prótese têm incidência de $1,1 \%{ }^{17}$, as ritidoplastias, de $2,8 \%$, com necessidade de abordagem cirúrgica, e de $1,7 \%$, sem necessidade de drenagem ${ }^{18}$. Nas abdominoplastias, a incidência é de $3 \%{ }^{19}$. Em relação à incidência de hematomas, os dados da literatura também foram mais elevados que os achados em nosso estudo, tanto antes quanto após a introdução do protocolo. Assim como a incidência de TEV, também ocorreu redução nas taxas de hematoma após o início do protocolo, embora tenha sido introduzido neste mesmo período o controle mais rigoroso da pressão arterial, o que pode ter criado um viés no resultado desta análise.

\section{CONCLUSÃO}

O protocolo de prevenção de TEV utilizado na Clínica Ivo Pitanguy está em uso desde maio de 2007; este tem se mostrado um instrumento de grande valia e de utilização simples e efetiva. No período estudado após a introdução do protocolo, não ocorreu nenhum caso de TVP ou TEP, num total de 711 pacientes operados e a incidência de hematomas com necessidade de revisão em centro cirúrgico foi reduzida.

\section{REFERÊNCIAS}

1. Davison SP, Venturi ML, Attinger CE, Baker SB, Spear SL. Prevention of venous thromboembolism in the plastic surgery patient. Plast Reconstr Surg. 2004;114(3):43E-51E.

2. Selby R, Geerts W. Prevention of venous thromboembolism: consensus, controversies, and challenges. Hematology Am Soc Hematol Educ Program. 2009;286-92.

3. Patronella CK, Ruiz-Razura A, Newall G, Mentz HA, Arango ML, Assavapokee T, et al. Thromboembolism in high-risk aesthetic surgery: experience with 17 patients in a review of 3871 consecutive cases. Aesthet Surg J. 2008;28(6):648-55.

4. Anger J, Baruzzi ACA, Knobel E. A prevention protocol for deep vein thrombosis in plastic surgery. Rev Bras Cir Plást. 2003;18(1):51-4.

5. Caprini JA, Arcelus JI, Reyna JJ. Effective risk stratification of surgical and nonsurgical patients for venous thromboembolic disease. Semin Hematol. 2001;38(2 Suppl 5):12-9.

6. Young VL, Watson ME. The need for venous thromboembolism (VTE) prophylaxis in plastic surgery. Aesthet Surg J. 2006;26(2):157-75.

7. Rosendaal FR. Venous thrombosis: the role of genes, environment, and behavior. Hematology Am Soc Hematol Educ Program. 2005;1-12.

8. Kroegel C, Reissig A. Principle mechanisms underlying venous thromboembolism: epidemiology, risk factors, pathophysiology and pathogenesis. Respiration. 2003;70(1):7-30.

9. Lanfranchi Jr A, Sardinha WE, Silvestre JMS, Palma O, Filho DMF, Silva MAF. Embolia pulmonar em necropsias no Hospital Universitário Regional do Norte do Paraná. Incidência em 10 anos e correlação clínico-anátomo-patológico. Cir Vasc Angiol. 1995;11(Supl 1):12.

10. Miszkiewicz K, Perreault I, Landes G, Harris PG, Sampalis JS, Dionyssopoulos A, et al. Venous thromboembolism in plastic surgery: incidence, current practice and recommendations. J Plast Reconstr Aesthet Surg. 2009;62(5):580-8.

11. McDevitt NB. Deep vein thrombosis prophylaxis. American Society of Plastic and Reconstructive Surgeons. Plast Reconstr Surg. 1999;104(6):1923-8.

12. Sellam P, Trevidic P. The thromboembolic risk in abdominal plastic surgery. A randomized statistical study of 190 cases. Ann Chir Plast Esthet. 1999;44(5):545-8.

13. Voss SC, Sharp HC, Scott JR. Abdominoplasty combined with gynecologic surgical procedures. Obstet Gynecol. 1986;67(2):181-5.

14. Virchow R. Thrombosis and emboli (1846-1856). Canton:Science History Publications; 1998.

15. Reinisch JF, Bresnick SD, Walker JW, Rosso RF. Deep venous thrombosis and pulmonary embolus after face lift: a study of incidence and prophylaxis. Plast Reconstr Surg. 2001;107(6):1570-5.

16. Aly AS, Cram AE, Chao M, Pang J, McKeon M. Belt lipectomy for circumferential truncal excess: the University of Iowa experience. Plast Reconstr Surg 2003;111(1):398-413.

17. Hvilsom GB, Hölmich LR, Henriksen TF, Lipworth L, McLaughlin JK, Friis S. Local complications after cosmetic breast augmentation: results from the Danish Registry for Plastic Surgery of the breast. Plast Reconstr Surg. 2009;124(3):919-25.

18. Jones BM, Grover R. Avoiding hematoma in cervicofacial rhytidectomy: a personal 8-year quest. Reviewing 910 patients. Plast Reconstr Surg. 2004;113(1):381-7.

19. Stewart KJ, Stewart DA, Coghlan B, Harrison DH, Jones BM, Waterhouse N. Complications of 278 consecutive abdominoplasties. J Plast Reconstr Aesthet Surg. 2006;59(11):1152-5. 